\title{
Foreword
}

\section{The Patient with}

Multimorbidities: Does $1+1$

Always Simply Equal 2?

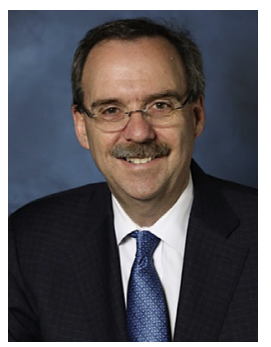

Lee A. Fleisher, MD, FACC, FAHA

Consulting Editor

Over the past several decades, anesthesiologists, surgeons, and others have developed strategies to care for the patient with medical conditions. These have taken the form of review papers or formal Guidelines like those produced by the American Heart Association/American College of Cardiology. Yet patients frequently have more than one medical condition, and some of the conditions have unique interactions or require contrary management approaches. In such circumstances, the simple addition of the recommendations may not be simple arithmetic. In this issue of Anesthesiology Clinics, the authors have evaluated some unique combinations of medical conditions that anesthesiologists might face, and provide outstanding advice on management.

This issue was proposed by a leader in thought in this arena and previous editor on this topic, Stanley H. Rosenbaum, MA, MD. Dr Rosenbaum is a Professor of Anesthesiology, Internal Medicine and Surgery at the Yale School of Medicine. He is the Vice-Chair for Academic Affairs and the Director of the Division of Perioperative and Adult Anesthesia within the Department of Anesthesiology. He received the Lifetime Achievement Award from the Society of Critical Care Anesthesiologists in 2011. He is joined by Robert B. Schonberger, MD, MHS, a cardiac anesthesiologist and clinical informatics researcher at the Yale School of Medicine, where he is an Assistant Professor of Anesthesiology. Dr Schonberger is an $\mathrm{NIH}$-funded researcher and has focused on the integration of surgical care and long-term cardiovascular risk reduction, the analysis of bias within large administrative datasets, and new techniques to minimize gaseous microemboli during extracorporeal support. Together, they have edited 
an issue that provides a guide to the current state-of-the-art, science, and care around the issues of the medically complex patient.

Lee A. Fleisher, MD, FACC, FAHA Perelman School of Medicine University of Pennsylvania 3400 Spruce Street, Dulles 680

Philadelphia, PA 19104, USA

E-mail address:

Lee.Fleisher@uphs.upenn.edu 